\title{
DETECTION OF SINGULARITIES USING SEGMENT APPROXIMATION
}

\author{
R. GROTHMANN AND H. N. MHASKAR
}

\begin{abstract}
We discuss best segment approximation (with free knots) by polynomials to piecewise analytic functions on a real interval. It is shown that, if the degree of the polynomials tends to infinity and the number of knots is the same as the number of singularities of the function, then the optimal knots converge geometrically fast to the singularities. When the degree is held fixed and the number of knots tends to infinity, we study the asymptotic distribution of the optimal knots.
\end{abstract}

\section{INTRODUCTION AND MAIN RESULTS}

The notion of segment approximation can be described in a very general way as follows. Let $L$ be a functional that associates with each continuous $f:[-1,1] \rightarrow \mathbb{R}$ and each subinterval $[a, b] \subseteq[-1,1]$ a nonnegative number $L(f,[a, b])$. Usually $L$ describes some constructive property of $f$ on $[a, b]$ such as the modulus of continuity of $f$ on $[a, b]$, the degree of approximation of $f$ by polynomials of a fixed degree on $[a, b]$, etc.

A partition

$$
-1=x_{0}<x_{1}<\cdots<x_{k+1}=1
$$

is called a leveled partition if

$$
L\left(f,\left[x_{i}, x_{i+1}\right]\right)=L\left(f,\left[x_{j}, x_{j+1}\right]\right), \quad 0 \leq i, j \leq k,
$$

and an optimal partition if

$$
\max _{0 \leq i \leq k} L\left(f,\left[x_{i}, x_{i+1}\right]\right) \leq \max _{0 \leq i \leq k} L\left(f,\left[z_{i}, z_{i+1}\right]\right)
$$

for all partitions $-1=z_{0}<z_{1}<\cdots<z_{k+1}=1$. The problem of segment approximation consists of determining an optimal partition $\left\{x_{i}\right\}$ and the value

$$
\max _{0 \leq i \leq k} L\left(f,\left[x_{i}, x_{i+1}\right]\right)
$$

for this partition. It is easy to see that all leveled partitions are optimal if $L$ is monotone on the intervals, i.e.,

$$
L\left(f, I_{1}\right) \leq L\left(f, I_{2}\right) \text { for all intervals } I_{1} \subseteq I_{2} \subseteq[-1,1] .
$$

In $[4,5]$, one can find efficient algorithms to determine the leveled and optimal partitions for such functionals. However, the existence of a leveled partition is guaranteed by the following theorem for a fairly large class of functionals $L$.

Received by the editor October 25, 1991.

1991 Mathematics Subject Classification. Primary 41A10, 41A15. 
Theorem 1.1. Let $L:[-1,1] \times[-1,1] \rightarrow[0, \infty)$ be continuous and $L(a, a)=$ 0 for $a \in[-1,1]$. Then for each $k \geq 1$ there exists a partition $-1=x_{0}<$ $x_{1}<\cdots<x_{k+1}=1$ such that

$$
L\left(x_{i}, x_{i+1}\right)=L\left(x_{j}, x_{j+1}\right), \quad 0 \leq i, j \leq k .
$$

In this paper, we are mainly interested in the following functionals. For an integer $n \geq 0, \Pi_{n}$ will denote the class of all real polynomials of degree at most $n$. For $n \geq 0$ we define the functional $d_{n}$ as follows. If $[a, b] \subseteq \mathbb{R}$ and $f \in C[a, b]$, then

$$
d_{n}(f,[a, b]):=\min _{p \in \Pi_{n}}\|f-p\|_{[a, b]},
$$

where $\|\cdot\|$ denotes the supremum norm on $[a, b]$. Next, for $n \geq 1$ and $[a, b] \subseteq \mathbb{R}$, let

$$
t_{j, n}:=t_{j, n, a, b}:=\frac{a+b}{2}+\frac{b-a}{2} \cos \left(\frac{2 j-1}{2 n} \pi\right), \quad j=1, \ldots, n .
$$

For $f \in C[a, b]$, let $L_{n}(f,[a, b]) \in \Pi_{n-1}$ be the polynomial satisfying

$$
L_{n}(f,[a, b])\left(t_{j, n}\right)=f\left(t_{j, n}\right), \quad 1 \leq j \leq n .
$$

We define the functional $\tilde{d}_{n}$ by

$$
\tilde{d}_{n}(f,[a, b]):=\left\|f-L_{n}(f,[a, b])\right\|_{[a, b]} .
$$

Of course, $\tilde{d}_{n}$ is much easier to compute than $d_{n}$. Furthermore, practical experience shows that the algorithm in [5] converges to a leveled partition for the functionals $\tilde{d}_{n}$ in (1.4).

The following theorem demonstrates that the leveled partitions corresponding to the functionals (1.2) and (1.4) can be used to detect certain singularities of $f$.

Theorem 1.2. Let $f \in C[a, b]$ be piecewise analytic, i.e., there is a partition

$$
-1=y_{0}<y_{1}<\cdots<y_{k+1}=1,
$$

such that for each integer $i, 0 \leq i \leq k, f_{i}:=\left.f\right|_{\left[y_{i}, y_{i+1}\right]}$ has an analytic continuation to a neighborhood of $\left[y_{i}, y_{i+1}\right]$, but does not extend to an analytic function on any neighborhood of any $y_{i}, i=1, \ldots, k$. Let, for each integer $n \geq 1$,

$$
-1=x_{n, 0}<x_{n, 1}<\cdots<x_{n, k+1}=1
$$

be a leveled partition for $f$ corresponding to $d_{n}$ (respectively $\tilde{d}_{n}$ ). Then, for each integer $i, 1 \leq i \leq k, \lim _{n \rightarrow \infty} x_{n, i}=y_{i}$. In fact,

$$
\limsup _{n \rightarrow \infty} \sup _{1 \leq i \leq k}\left|x_{n, i}-y_{i}\right|^{1 / n}<1 \text {. }
$$

The proof of Theorem 1.2 depends on the characterization of analytic functions due to Bernstein and a theorem of Hasson on derivatives of polynomials of best approximation. When more information is available about the function, for example when the functions $f_{i}$ are entire functions of given order and type, the rates of convergence in (1.5) can be strengthened accordingly (see Corollary 3.1).

The following counterexample presents a case where the internal knot does not converge to the internal singularity of the function $f$. However, in this case the function is not differentiable in the first segment interval. 
Example 1.3. Let $k=1$ and $-1<x_{n}:=x_{1, n}<1$ be a leveled partition for the following function $f$ corresponding to the functional $d_{n}$ :

$$
f(x)= \begin{cases}\sqrt{x+1} & \text { for }-1 \leq x \leq 0 \\ 1+x / 2 & \text { for } 0<x \leq 1\end{cases}
$$

Then we claim that $x_{n}$ converges to -1 . Assume that $x_{n}>-1+\varepsilon$ for $n \in \Lambda$, where $\Lambda$ is a subsequence of $\mathbb{N}$ and $\varepsilon>0$. By a theorem of Bernstein [1], for $\varepsilon>0$,

$$
\lim _{n \rightarrow \infty, n \in \Lambda} n d_{n}(f,[-1,-1+\varepsilon])=C \sqrt{\varepsilon}
$$

Thus,

$$
\limsup _{n \rightarrow \infty} n d_{n}\left(f,\left[-1, x_{n}\right]\right) \geq C_{1}>0 .
$$

On the other hand, $f$ is continuously differentiable on $[-1+\varepsilon, 1]$, and $f^{\prime}$ satisfies a Lipschitz condition of order 1. By a theorem of Jackson [6],

$$
\limsup _{n \rightarrow \infty} n d_{n}\left(f,\left[x_{n}, 1\right]\right)=0 \text {. }
$$

Since, by the definition of $x_{n}, d_{n}\left(f,\left[-1, x_{n}\right]\right)=d\left(f,\left[x_{n}, 1\right]\right)$ for all integers $n \geq 1$, this is a contradiction.

Next we consider the case when the degree of polynomials stays fixed, but the number of knots tends to infinity. For any partition

$$
P:-1=z_{0}<z_{1}<\cdots<z_{k+1}=1,
$$

we define the measure $\tau_{P}$ by the formula

$$
\tau_{P}(A):=\frac{\left|\left\{j: z_{j} \in A\right\}\right|}{k+2}
$$

for Borel sets $A \subseteq[-1,1]$.

Theorem 1.4. Let $n \geq 0$ be a fixed integer, $f$ be $n+1$ times continuously differentiable on $[-1,1]$, and for an integer $k \geq 0$

$$
P_{k}:-1=\xi_{k, 0}<\xi_{k, 1}<\cdots<\xi_{k, k+1}=1
$$

be a leveled partition for $f$ corresponding to $d_{n}$ (respectively $\tilde{d}_{n}$ ). Furthermore, we assume that $f$ is not a polynomial in $\Pi_{n}$ on any subinterval $[-1,1]$. Set $\lambda:=\int_{-1}^{1}\left|f^{(n+1)}(t)\right|^{1 / n} d t$ and $E_{k}:=d_{n}\left(f,\left[\xi_{k, 0}, \xi_{k, 1}\right]\right.$ ) (respectively $E_{k}:=$ $\left.\tilde{d}_{n}\left(f,\left[\xi_{k, 0}, \xi_{k, 1}\right]\right)\right)$. Then

$$
\lim _{k \rightarrow \infty}(k+1)\left[(n+1) ! 2^{n} E_{k}\right]^{1 / n}=\lambda
$$

and, for any $[a, b] \subseteq[-1,1]$,

$$
\lim _{k \rightarrow \infty} \tau_{P_{k}}([a, b])=\frac{1}{\lambda} \int_{a}^{b}\left|f^{(n+1)}(t)\right|^{1 / n} d t .
$$

\section{NUMERICAL RESULTS}

In our first example, we set

$$
f_{1}(x):=|\cos \pi(x-0.1)|, \quad x \in[-1,1],
$$


TABLE 1

\begin{tabular}{c|cccc|c}
\hline degree & $y_{0}$ & $y_{1}$ & $y_{2}$ & $y_{3}$ & error \\
\hline 3 & -1 & -0.3907 & 0.5899 & 1 & 0.026 \\
5 & -1 & -0.3998 & 0.5992 & 1 & 0.00059 \\
7 & -1 & -0.3999978 & 0.6000002 & 1 & 0.0000068 \\
\hline
\end{tabular}

TABLE 2

\begin{tabular}{c|ccc|c}
\hline degree & $y_{0}$ & $y_{1}$ & $y_{2}$ & error \\
\hline 3 & 0 & 0.057 & 1 & 0.01 \\
5 & 0 & 0.02 & 1 & 0.0039 \\
7 & 0 & 0.015 & 1 & 0.0025 \\
\hline
\end{tabular}

TABLE 3

\begin{tabular}{c|ccc|c}
\hline degree & $y_{0}$ & $y_{1}$ & $y_{2}$ & error \\
\hline 3 & 0.1 & 0.4035 & 1 & 0.00083 \\
5 & 0.1 & 0.2538 & 1 & 0.00000815 \\
7 & 0.1 & 0.2498 & 1 & 0.00000007 \\
\hline
\end{tabular}

so that the true singularities $y_{1}$ and $y_{2}$ are respectively -0.4 and 0.6 . The function $f_{1}$ is approximated with the help of an algorithm based on [5]. The results are summarized in Table 1 , where error means deviation of $f_{1}$ from its best segment approximation.

Next we choose the function

$$
f_{2}(x):= \begin{cases}\sqrt{x}, & 0 \leq x \leq 1 / 4, \\ 1 / 2 e^{2 x-1 / 2}, & 1 / 4 \leq x \leq 1 .\end{cases}
$$

First we approximate this function on $[0,1]$ with one internal knot (Table 2). As expected from Example 1.3 we have $y_{1} \rightarrow 0$.

Finally, we approximate $f_{2}$ on the interval $[0.1,1]$ with one internal knot (Table 3).

\section{Proofs}

Proof of Theorem 1.1. We may assume that for each partition (1.1) there is at least one $j \in\{1, \ldots, k+1\}$ with $L\left(x_{j-1}, x_{j}\right)>0$. For, otherwise, the 
theorem is already proved. Define

$$
M=\left\{\left(\delta_{1}, \ldots, \delta_{k+1}\right) \in \mathbb{R}^{k+1}: \sum_{i=1}^{k+1} \delta_{i}=2, \delta_{i} \geq 0 \text { for } i=1, \ldots, k+1\right\}
$$

and set

$$
S(d):=2 \frac{\left(p_{1}, \ldots, p_{k+1}\right)}{\sum_{i=1}^{k+1} p_{i}}
$$

for $d=\left(\delta_{1}, \ldots, \delta_{k+1}\right) \in M$, where

$$
p_{i}:=L\left(-1+\sum_{j=1}^{i-1} \delta_{j},-1+\sum_{j=1}^{i} \delta_{j}\right) .
$$

Obviously, $S$ is a continuous operator, mapping $M$ to itself. It suffices to prove that there is a $d \in M$ with

$$
S(d)=\zeta:=\left(\frac{2}{k+1}, \ldots, \frac{2}{k+1}\right) .
$$

Assume the contrary. Then we define an operator $T: M \rightarrow M$ as follows. For $m \in M$ we construct the straight line $L_{m}$ which runs through $S(m)$ and $\zeta$. The intersection of $L_{m}$ with $M$ is a line segment with endpoints $p_{1}$ and $p_{2}$. Set $T(m)=p_{1}$ if $p_{1}$ is the endpoint such that $\zeta$ lies between $p_{1}$ and $S(m)$, and $p_{2}$ otherwise. Since $S(m) \neq \zeta$, the operator $T$ is well defined and continuous. Furthermore, $T$ maps $M$ to itself.

In view of Brower's fixed point theorem, $T$ has a fixed point $d=\left(\delta_{1}, \ldots\right.$, $\left.\delta_{k+1}\right) \in M$. By the construction of $T, \delta_{j}=0$ for a $j \in\{1, \ldots, k+1\}$. Let $S(d)=:\left(s_{1}, \ldots, s_{k+1}\right)$. Since $L(a, a)=0$ for all $a \in[-1,1]$, we see that $s_{j}=0$. The construction of $T$ then implies that the $j$ th component of $T(d)=d$ is not zero, i.e., $\delta_{j} \neq 0$. This contradiction proves that (3.1) holds for some $d \in M$.

Proof of Theorem 1.2. We observe that a piecewise analytic function cannot be infinitely many times differentiable on $[-1,1]$. For $1 \leq i \leq k$, let $r_{i}>0$ be the smallest integer such that $f^{\left(r_{i}\right)}\left(y_{i}+\right) \neq f^{\left(r_{i}\right)}\left(y_{i}-\right)$ and set

$$
\sigma:=\inf _{1 \leq i \leq k}\left|f^{\left(r_{i}\right)}\left(y_{i}+\right)-f^{\left(r_{i}\right)}\left(y_{i}-\right)\right|>0 .
$$

We now fix $i, 1 \leq i \leq k$. For integers $n \geq 0$, we set $\varepsilon_{i, n}:=\inf _{1 \leq l \leq k}\left|x_{n, l}-y_{i}\right|$, and we shall estimate $\sigma$ in terms of $\varepsilon_{i, n}$. For the sake of concreteness, we shall assume that $\left\{x_{n, l}\right\}$ is a leveled partition for $\tilde{d}_{n}$. The proof for the case of $d_{n}$ is similar and simpler.

Let $a_{j}<y_{j}<y_{j+1}<b_{j}$ be found so that $f_{j}$ can be extended to an analytic function on a neighborhood of $\left[a_{j}, b_{j}\right], j=0, \ldots, k$. Let

$$
D_{n}:=\max _{0 \leq j \leq k} d_{n}\left(f_{j},\left[a_{j}, b_{j}\right]\right)
$$

and, for any $j, 0 \leq j \leq k$,

$$
\widetilde{D}_{n}:=\tilde{d}_{n}\left(f,\left[x_{n, j}, x_{n, j+1}\right]\right) .
$$


We observe that at least one of the intervals $\left[x_{n, j}, x_{n, j+1}\right]$ is contained in an interval $\left[y_{l}, y_{l+1}\right], 0 \leq j, l \leq k$. Thus, it follows from a well-known result $[3$, Vol. III, p. 48] that

$$
\widetilde{D}_{n} \leq c \log n D_{n} .
$$

(Here and in the sequel, $c, c_{1}, \ldots$ will denote positive constants independent of $n$ and the function involved.)

Let

$$
P_{n}(t):= \begin{cases}L_{n}\left(f,\left[x_{n, j}, x_{n, j+1}\right]\right)(t), & x_{n, j} \leq t<x_{n, j+1}, 0 \leq j \leq k-1, \\ L_{n}\left(f,\left[x_{n, k}, 1\right]\right)(t), & x_{n, k} \leq t \leq 1 .\end{cases}
$$

Then, by (3.2),

$$
\left\|f-P_{n}\right\|_{[-1,1]} \leq c \log n D_{n} .
$$

Let $n \in \mathbb{N}$ such that $\varepsilon_{i, n}>0$. If $p_{n} \in \Pi_{n}$ denotes the polynomial of best approximation to $f_{i}$ on $\left[a_{i}, b_{i}\right]$, then by a theorem due to Hasson [2], we have

$$
\left\|p_{n}^{\left(r_{i}\right)}-f_{i}^{\left(r_{i}\right)}\right\|_{\left[y_{i}, y_{i+1}\right]} \leq c_{1} n^{r} D_{n} .
$$

Let $K_{i}:=\left[y_{i}, y_{i}+\varepsilon_{i, n}\right]$. Then $P_{n}$ is a polynomial on $K_{i}$. Using the Markov inequality and (3.3), we obtain

$$
\left\|P_{n}^{\left(r_{i}\right)}-p_{n}^{\left(r_{i}\right)}\right\|_{K_{i}} \leq \frac{c_{2} n^{2 r_{i}}}{\varepsilon_{i, n}^{r_{i}}}\left\|P_{n}-p_{n}\right\|_{K_{i}} \leq \frac{c_{3} n^{2 r_{i}} \log n D_{n}}{\varepsilon_{i, n}^{r_{i}}} .
$$

Hence, (3.4) implies

$$
\left|P_{n}^{\left(r_{i}\right)}\left(y_{i}\right)-f^{\left(r_{i}\right)}\left(y_{i}+\right)\right|=\left|P_{n}^{\left(r_{i}\right)}\left(y_{i}\right)-f_{i}^{\left(r_{i}\right)}\left(y_{i}\right)\right| \leq \frac{c_{4} n^{2 r_{i}} \log n D_{n}}{\varepsilon_{i, n}^{r_{i}}} .
$$

Estimating $\left|P_{n}^{\left(r_{i}\right)}\left(y_{i}\right)-f^{\left(r_{i}\right)}\left(y_{i}-\right)\right|$ in the same manner, we see that

$$
\sigma \leq\left|f^{\left(r_{i}\right)}\left(y_{i}+\right)-f^{\left(r_{i}\right)}\left(y_{i}-\right)\right| \leq c_{5} \frac{n^{2 r_{i}} \log n D_{n}}{\varepsilon_{i, n}^{r_{i}}} .
$$

Hence,

$$
\varepsilon_{i, n} \leq c_{6} \frac{n^{2}\left(\log n D_{n}\right)^{1 / r_{i}}}{\sigma^{1 / r_{i}}} .
$$

This estimate is valid for all $n \in \mathbb{N}$ and $1 \leq i \leq k$. Since $D_{n} \rightarrow 0$ exponentially fast, $\varepsilon_{i, n} \rightarrow 0$ exponentially fast, $1 \leq i \leq k$. However, this is only possible if $x_{n, i} \rightarrow y_{i}$ exponentially fast. This proves Theorem 1.2.

It is clear from the proof that the operator $L_{n}$ can be replaced by a wide class of operators. As long as $\log n$ in (3.3) is replaced by constants $C_{n}$ such that $\lim _{\sup _{n \rightarrow \infty}} C_{n}^{1 / n}=1$, the theorem remains true.

Furthermore, we note the following corollaries of the proof of Theorem 1.2.

Corollary 3.1. If each $f_{i}$ is an entire function of order 1 and finite exponential type $\tau, 0 \leq i \leq k$, then for any $\delta>0$, using the notation of the proof of Theorem 1.2,

$$
\varepsilon_{i, n} \leq \frac{c(\tau+\delta)^{n}}{\sigma^{1 / r_{i} n !}}
$$


Corollary 3.2. Let $m \geq 2$ and $k \geq 1$ be integers, $\left\{y_{i}\right\}_{i=0}^{k+1}$ be as in Theorem 1.2 , and $f:[-1,1] \rightarrow \mathbb{R}$ be m-times continuously differentiable on each interval $\left[y_{i}, y_{i+1}\right], 0 \leq i \leq k$, but not $([m / 2]-1)$-times continuously differentiable in the points $y_{i}, i=1, \ldots, k$. Let $\left\{x_{n, i}\right\}_{i=0}^{k+1}$ be the leveled partitions for $f$ as in Theorem 1.2. Then $\lim _{n \rightarrow \infty} x_{n, i}=y_{i}$ for $1 \leq i \leq k$.

Proof of Theorem 1.4. As in the proof of Theorem 1.2, we consider only the case of the functional $d_{n}$ : the case of $\tilde{d}_{n}$ is simpler. Denote by $E_{k}$ the error of approximation with $k$ internal knots. Since

$$
\limsup _{k \rightarrow \infty} \sup _{0 \leq \eta \leq 2 /(k+1),-1 \leq x \leq 1-\eta} d_{n}(f,[x, x+\eta])=0
$$

and at least one of the intervals $\left[\xi_{k, j}, \xi_{k, j+1}\right]$ has length not exceeding $2 /(k+1), E_{k}$ tends to 0 as $k \rightarrow \infty$. Set $\varepsilon_{k, \nu}:=\xi_{k, \nu+1}-\xi_{k, \nu}, \nu=0, \ldots, k$.

We claim that

$$
\lim _{k \rightarrow \infty} \max _{0 \leq \nu \leq k} \varepsilon_{k, \nu}=0 .
$$

If not, a compactness argument shows that there is a subinterval $I=[\alpha, \beta]$, $\alpha<\beta$, of $[-1,1]$ such that

$$
I \subseteq\left[\xi_{k, \nu(k)}, \xi_{k, \nu(k)+1}\right], \quad k=k_{1}, k_{2}, \ldots,
$$

for a subsequence $k_{1}<k_{2}<\cdots$ of $\mathbb{N}$. Since $E_{k}$ tends to 0 , the function $f$ must be a polynomial of degree $n$ on $I$. This contradiction proves our claim.

Let

$$
I_{k, \nu}:=\left[\xi_{k, \nu}, \xi_{k, \nu+1}\right], \quad k \in \mathbb{N}, \nu=0, \ldots, k,
$$

and define $p_{k, \nu} \in \Pi_{n}$ to be the polynomial of best approximation to $f$ on $I_{k, \nu}$. Interpolating in the roots of the $n$ th-degree Chebyshev polynomial scaled to the interval $I_{\nu}$, a well-known formula for the error of interpolation [3, Vol. III, p. 10] implies that

$$
E_{k} \leq \max _{I_{\nu}} \frac{\left|f^{(n+1)}\right|}{(n+1) !} \frac{\varepsilon_{k, \nu}^{n}}{2^{n-1}} .
$$

On the other hand, in view of the Chebyshev alternation theorem, $p_{k, \nu}$ interpolates $f$ on at least $n$ distinct points $\tilde{t}_{k, \nu, 1}<\cdots<\tilde{t}_{k, \nu, n}$ in $I_{\nu}$. Hence,

$$
f(x)-p_{k, \nu}(x)=\frac{f^{n+1}\left(\xi_{x}\right)}{(n+1) !} \omega_{k, \nu}(x),
$$

where $\xi_{x}$ is a point in $I_{k, \nu}$ and $\omega_{k, \nu}(x)=\prod_{\mu=1}^{n}\left(x-\tilde{t}_{k, \nu, \mu}\right)$. Since the Chebyshev polynomial (scaled to $I_{k, \nu}$ ) has the minimal norm among all monic polynomials in $\Pi_{n}$, we have

$$
\max _{t \in I_{k, \nu}}\left|\omega_{k, \nu}(t)\right|=:\left|\omega\left(s_{k, \nu}\right)\right| \geq \frac{\varepsilon_{k, \nu}^{n}}{2^{n-1}} .
$$

Hence,

$$
\begin{aligned}
E_{k} & \geq\left|\left(f-p_{k, \nu}\right)\left(s_{k, \nu}\right)\right| \geq \frac{\min _{t \in I_{k, \nu}}\left|f^{(n+1)}\right|}{(n+1) !} \max _{t \in I_{k, \nu}}\left|\omega_{k, \nu}(t)\right| \\
& \geq \frac{\min _{t \in I_{k, \nu}}\left|f^{(n+1)}\right|}{(n+1) !} \frac{\varepsilon_{k, \nu}^{n}}{2^{n-1}} .
\end{aligned}
$$


Setting $c:=\left((n+1) ! 2^{n-1}\right)^{1 / n}$, we get

$$
\min _{t \in I_{\nu}}\left|f^{(n+1)}(t)\right|^{1 / n} \varepsilon_{k, \nu} \leq c E_{k}^{1 / n} \leq \max _{t \in I_{\nu}}\left|f^{(n+1)}(t)\right|^{1 / n} \varepsilon_{k, \nu} .
$$

By summing over $\nu=0, \ldots, k$, it follows from the Riemann integrability of $\left|f^{(n+1)}\right|^{1 / n}$ that

$$
\lim _{k \rightarrow \infty}(k+1) c E_{k}^{1 / n}=\int_{-1}^{1}\left|f^{(n+1)}(t)\right|^{1 / n} d t=\lambda .
$$

Fix $-1 \leq \alpha \leq \beta \leq 1$ and let $\tau$ be any weak limit point of the sequence of unit measures $\left(\tau_{k}\right)$. Again using the Riemann integrability of $\left|f^{(n+1)}\right|^{1 / n}$, we have

$$
\tau([\alpha, \beta]) \lambda=\lim _{k \rightarrow \infty}(k+1) \tau_{k}([\alpha, \beta]) c E_{k}^{1 / n}=\int_{\alpha}^{\beta}\left|f^{(n+1)}(t)\right|^{1 / n} d t .
$$

This proves the theorem.

\section{ACKNOWLEDGMENT}

The work of H. N. Mhaskar was supported, in part, by the Katholische Universität, Eichstätt, during his visit there in Summer, 1990. The authors thank Professor H.-P. Blatt for bringing the papers [4] and [5] to their attention.

\section{BIBLIOGRAPHY}

1. S. N. Bernstein, Sur la valeur asymptotique de la meilleure approximation de $|x|$, Acta Math. 37 (1913), 1-57.

2. M. Hasson, Derivatives of the algebraic polynomials of best approximation, J. Approx. Theory 29 (1980), 91-102.

3. I. P. Natanson, Constructive theory of functions, Vols. I-III, Ungar, New York, 1964.

4. G. Nürnberger, M. Sommer, and H. Strauss, An algorithm for segment approximation, Numer. Math. 48 (1986), 463-477.

5. G. Meinardus, G. Nürnberger, M. Sommer, and H. Strauss, Algorithms for piecewise polynomials and splines with free knots, Math. Comp. 53 (1989), 235-247.

6. A. F. Timan, Theory of approximation of functions of a real variable, Pergamon Press, 1963.

Mathematisch-Geographische Fakultät, Katholische Universität Eichstätt, OstenSTR. 18, D-8078 EichSTÄTt, GERMANY

Department of Mathematics, California State University, Los Angeles, California 90032 